EESTI NSV TEADUSTE AKADEEMIA TOIMETISED. IX KÖIDE FOOSIKALIS-MATEMAATILISTE JA TEHNILISTE TEADUSTE SEERIA, 1960, NR. 4

ИЗВЕСТИЯ АКАДЕМИИ НАУК ЭСТОНСКОИ ССР. ТОМ ІХ СЕРИЯ ФИЗИКО-МАТЕМАТИЧЕСКИХ И ТЕХНИЧЕСКИХ НАУК. 1960, № 4

\title{
ОБ ОДНОМ ОБОБЩЕНИИ МЕТОДА НЬЮТОНА
}

\section{л. Кивистик}

В настоящей статье рассматриваются два класса итерационных методов для решения операторного уравнения $P(x)=0$ и доказывается сходимость этих методов. Для оценки погрешностей выводятся формулы, уточняющие в случае метода Ньютона оценки, полученные Л. В. Канторовичем ['] и С. Ю. Ульмом [].

1. Пусть $P(x)$ есть нелинейный, дважды дифференцируемый (в смысле Фреше) оператор из банахова пространства $X$ в пространство $Y$ того же типа. Для решения уравнения

$$
P(x)=0
$$

рассмотрим итерационный метод

$$
x_{n+1}=x_{n}-\alpha_{n} \Gamma\left(x_{n}\right) P\left(x_{n}\right),
$$

где $\Gamma(x)=\left[P^{\prime}(x)\right]^{-1}$ и $\alpha_{n}$ - вещественные числа из промежутка $(0,2)$. В частных случаях мы налагаем на $\alpha_{n}$ еще некоторые дополнительные ограничения.

Если $\alpha_{n}=1(n=0,1, \ldots)$, то из (2) получается известный метод Ньютона, а если $0<\alpha_{n}=\alpha<1-$ обобщенный процесс Д. А. Граве (см. [']). О сходимости этих методов Л. В. Канторович [1] доказал теоремы, которые (если их объединить) можно сформулировать следующим образом:

Т ео рем а А. Пусть выполнены условия:

$1^{\circ}$ существует обратный оператор $\Gamma\left(x_{0}\right)=\left[P^{\prime}\left(x_{0}\right)\right]^{-1}, \quad$ причем $\left\|\Gamma\left(x_{0}\right)\right\| \leqslant B_{0}$;

$2^{\circ}\left\|\Gamma\left(x_{0}\right) P\left(x_{0}\right)\right\| \leqslant \eta_{0} ;$

$3^{\circ}$ для всех $x \in S\left(x_{0}, r\right)$ *, где

$$
r=N\left(h_{0}\right) \eta_{0}=\frac{1-\sqrt{1-2 h_{0}}}{h_{0}} \eta_{0},
$$

имеет место неравенство $\left\|P^{\prime \prime}(x)\right\| \leqslant K$;

$$
4^{\circ} h_{0}=B_{0} K \eta_{0} \leqslant \frac{1}{2} .
$$

- Символом $S\left(x_{0}, r\right)$ мы обозначаем сферу $\left\|x-x_{0}\right\| \leqslant r$. 
Тогда уравнение (1) имеет в сфере $S\left(x_{0}, r\right)$ решение $x^{*}$, и последовательные приближения процесса (2), где $0<\alpha_{n}=\alpha \leqslant 1$, сходятся $\kappa$ нему. Если $\alpha=1$, то имеет место оценка

$$
\left\|x^{*}-x_{n}\right\| \leqslant \frac{1}{2^{n-1}}\left(2 h_{0}\right)^{2^{\prime \prime}-1} \eta_{0} .
$$

Ниже мы распространим эту теорему на случай, когда $\alpha_{n}$ произвольные вещественные числа из полуинтервала $(0,1]$, причем inf $\alpha_{n}=\alpha>0$ (в дальнейшем мы рассмотрим случай, когда $0<\alpha_{n}<2$ ). Но прежде всего остановимся на вопросе об апостериорной оценке погрешностей итерационных методов.

2. Формулы для апостериорной оценки погрешностей итерационных методов решения уравнения (1) имеют практическое значение даже в том случае, когда известны формулы для априорной оценки. Это объясняется, во-первых, тем, что апостериорные оценки обычно значияельно более точны, так как они зависят от структуры оператора $P$ в самой оцениваемой точке $x_{n}$ (или в точке $x_{n-1}$ ), в то время как априорные оценки зависят только от структуры оператора в точке $x_{0}$. Во-вторых, апостериорные оценки дают правильные результаты и в том случае, когда некоторые промежуточные вычисления ошибочны (в известных пределах) или когда последовательные приближения вычислены по итерационной формуле п р и бл и женн н. Следует еще отметить, что если вычисления осуществляются при помощи быстродействующих электронных счетных машин, то почти всегда целесообразнее пользоваться апостериорными оценками.

Пусть

$$
x_{n+1}=x_{n}+Q_{n}\left(x_{n}\right)
$$

есть некоторая итерационная формула для нахождения последовательных приближений к решению $x^{*}$ уравнения (1), где $Q_{n}$-операторы, характеризующие рассматриваемый метод. Пусть известно, что сходи мость метода (4) доказывается тем путем, что, предполагая выполнение некоторых (достаточных для сходимости) условий при $x=x_{0}$, выводят, что эти условия остаются в силе при всех $x_{n+1}(n=0,1, \ldots)$.

В ходе доказательства получается некоторая (априорная) оценка

$$
\left\|x^{*}-x_{0}\right\| \leqslant \Phi\left(x_{0}\right),
$$

где $\Phi\left(x_{0}\right)$ вычисляется при помощи непосредственной оценки норм известных, зависящих от $x$, операторов или значений операторов при $x=x_{0}$.

Так как любое $x_{n}$ можно считать «начальным приближением», то справедливо также неравенство

$$
\left\|x^{*}-x_{n}\right\| \leqslant \Phi\left(x_{n}\right),
$$

где $\Phi\left(x_{n}\right)$ вычисляется при помощи непосредственной оценки соответствующих норм при $x=x_{n}$. Неравенством (5) можно обычно пользоваться как апостериорной оценкой погрешностей метода (4).

Отметим, что этим путем можно получить апостериорные оценки почти для всех известных итерационных методов, если их априорные оценки известны. В частности, такие оценки можно получить для методов, рассмотренных в работах $\left[{ }^{1-5}\right]$. Например, в случае метода Ньютона

* Теоремы сходимости методов ньютоновского типа обычно так и доказываются. 
имеет место оценка

$$
\left\|x^{*}-x_{n}\right\| \leqslant 2\left\|\Gamma\left(x_{n}\right) P\left(x_{n}\right)\right\|,
$$

так как из теоремы А вытекает неравенство $\left\|x^{*}-x_{0}\right\| \leqslant 2\left\|\Gamma\left(x_{0}\right) P\left(x_{0}\right)\right\|$.

Олисанный способ получения апостериорных оценок мы используем в последующих пунктах.

3. Рассмотрим вопрос о сходимости метода (2).

Т еорема 1. Пусть выполнены условия $1^{\circ}-4^{\circ}$ теоремы $A$ и пусть $0<\alpha_{n} \leqslant 1, \alpha=\inf \alpha_{n}>0$. Тогда уравнение (1) имеет в сфере $S\left(x_{0}, r\right)$ решение $x^{*}, \kappa$ которому сходится последовательность $\left\{x_{n}\right\}$, полу. ченная методом (2), и имеют место оценки

$$
\left\|x^{*}-x_{n}\right\| \leqslant N\left(\bar{h}_{n}\right)\left\|\Gamma\left(x_{n}\right) P\left(x_{n}\right)\right\| \leqslant N\left(h_{0}\right) \eta_{0} \cdot q^{n},
$$

гेe

$$
\bar{h}_{n}=\left\|\Gamma\left(x_{n}\right)\right\| K\left\|\Gamma\left(x_{n}\right) P\left(x_{n}\right)\right\| \quad u \quad q=\frac{1-\alpha+\frac{1}{2} a^{2} h_{0}}{1-a h_{0}} .
$$

Доказ а тельс т в в общих чертах совпадает с доказательством теоремы А (см. $\left.\left[{ }^{1}\right]\right)$. Изложим здесь только некоторые моменты, существенные в дальнейшем.

Условия $1^{\circ}-4^{\circ}$ остаются выполненными, если $x_{0}$ заменить на $x_{n+1}$ $(n=0,1, \ldots)$. В самом деле, можно показать, что:

1) существует оператор $\Gamma\left(x_{n+1}\right)$, причем

$$
\left\|\Gamma\left(x_{n+1}\right)\right\| \leqslant \frac{B_{n}}{1-a_{n} h_{n}}=B_{n+1} ;
$$

2) $\left\|\Gamma\left(x_{n+1}\right) P\left(x_{n+1}\right)\right\| \leqslant \frac{1-a_{n}+\frac{1}{2} a_{n}^{2} h_{n}}{1-a_{n} h_{n}} \eta_{n}=\eta_{n+1}$;

3) при всех $x \in S\left(x_{n+1}, r_{n+1}\right)$, где $r_{n+1}=N\left(h_{n+1}\right) \eta_{n+1}$, справедливо неравенство $\left\|P^{\prime \prime}(x)\right\| \leqslant K\left(\right.$ так как $\left.S\left(x_{n+1}, r_{n+1}\right) \subset S\left(x_{0}, r\right)\right)$;

4) $h_{n+1}=B_{n+1} K \eta_{n+1} \leqslant h_{0} \leqslant \frac{1}{2}$, причем

$$
h_{n+1}=\frac{1-a_{n}+\frac{1}{2} a_{n}^{2} h_{n}}{\left(1-a_{n} h_{n}\right)^{2}} h_{n}
$$

Используя рекуррентные соотношения (8) и (7), легко нелосредственно проверить, что имеет место тождество

$$
N\left(h_{k+1}\right) \eta_{k+1}=N\left(h_{k}\right) \eta_{k}-\alpha_{k} \eta_{k}
$$

При помощи (2), (7) и (9) получим

$$
\begin{aligned}
& \left\|x_{n+p}-x_{n}\right\| \leqslant \alpha_{n} \eta_{n}+\alpha_{n+1} \eta_{n+1}+\ldots+\alpha_{n+p-1} \eta_{n+p-1}= \\
& \quad=N\left(h_{n}\right) \eta_{n}-N\left(h_{n+p}\right) \eta_{n+p}<N\left(h_{n}\right) \eta_{n} .
\end{aligned}
$$


Из равенства (7) следует, что

$$
\eta_{n+1} \leqslant q \eta_{n} \leqslant q^{n+1} \eta_{0}
$$

Так как $1 \leqslant N(h) \leqslant 2\left(\right.$ если $\left.0 \leqslant h \leqslant \frac{1}{2}\right)$ и $q<1$ (в силу условия $4^{\circ}$ ), то из (10) и (11) следует, что существует $x^{*}=\lim _{n \rightarrow \infty} x_{n}$. Вместе с тем получим

$$
\left\|x^{*}-x_{n}\right\| \leqslant N\left(h_{n}\right) \eta_{n} \leqslant N\left(h_{0}\right) \eta_{0} q^{n},
$$

т. е. вторую часть оценки (6). В силу сказанного в пункте 2 , отсюда следует и апостериорная оценка.

Так как $\frac{1}{a_{n}} \leqslant \frac{1}{a}<+\infty$ и $\lim _{n \rightarrow \infty}\left\|P^{\prime}\left(x_{n}\right)\right\|<+\infty$, а $\lim _{n \rightarrow \infty}\left\|x_{n+1}-x_{n}\right\|=0$, то переход к пределу в тождестве

$$
P\left(x_{n}\right)+\frac{1}{a_{n}} P^{\prime}\left(x_{n}\right)\left(x_{n+1}-x_{n}\right)=0
$$

показывает, что $x^{*}$ является решением уравнения (1).

Пр имеч а ни е 1 . Применение апостериорной оценки $\left\|x^{*}-x_{n}\right\| \leqslant$ $\leqslant N\left(\bar{h}_{n}\right)\left\|\Gamma\left(x_{n}\right) P\left(x_{n}\right)\right\|=\frac{N\left(\bar{h}_{n}\right)}{e_{n}}\left\|x_{n+1}-x_{n}\right\| \quad$ фактически требует нахождения последующего приближения $x_{n+1}$. Приведем оценки, применение которых требует меньше вычислений.

Обозначим $\overline{h_{k}}=\left\|\Gamma\left(x_{k}\right)\right\| K\left\|\Gamma\left(x_{k}\right) P\left(x_{k}\right)\right\|$. Так как $\Gamma\left(x_{n}\right)=H_{n-1} \Gamma\left(x_{n-1}\right)$, где $H_{n-1}=\left[E-I\left(x_{n-1}\right)\left(P^{\prime}\left(x_{n-1}\right)-P^{\prime}\left(x_{n}\right)\right)\right]^{-1}(E-$ единичный оператор пространства $X)$, и $\left\|H_{n-1}\right\| \leqslant\left(1-\alpha_{n-1} \cdot \bar{h}_{n-1}\right)^{-1}$, то получим неравенство

$$
\bar{h}_{n} \leqslant \frac{1-a_{n-1}+\frac{1}{2} a_{n-1}^{2} \bar{h}_{n-1}}{\left(1-a_{n-1} \bar{h}_{n-1}\right)^{2}} \bar{h}_{n-1} .
$$

Учитывая, что $N(h)$ - возрастающая функция, нетрудно проверить, что имеет место неравенство

$$
N\left(\overline{h_{n}}\right) \leqslant\left(1-\alpha_{n-1} \bar{h}_{n-1}\right) N^{2}\left(\bar{h}_{n-1}\right) .
$$

Используя последнее неравенство, получим оценку

$$
\left\|x^{*}-x_{n}\right\| \leqslant N^{2}\left(\bar{h}_{n-1}\right)\left\|\Gamma\left(x_{n-1}\right) P\left(x_{n}\right)\right\|,
$$

предложенную в несколько иной форме Л. В. Канторовичем [ $\left.{ }^{1}\right]$ при $n=1$ для метода Ньютона $\left(\alpha_{n}=1, n=0,1, \ldots\right)$. Учитывая соотношение $(2)$, можем норму $\left\|\Gamma\left(x_{n-1}\right) P\left(x_{n}\right)\right\|$ в свою очередь оценить следующим образом:

$$
\left\|\Gamma\left(x_{n-1}\right) P\left(x_{n}\right)\right\| \leqslant\left(1-\alpha_{n-1}+\frac{1}{2} \alpha_{n-1}^{2} \cdot \bar{h}_{n-1}\right)\left\|\Gamma\left(x_{n-1}\right) P\left(x_{n-1}\right)\right\| .
$$


4. Пусть по-прежнему выполнены условия теоремы 1 , а постоянные $\alpha_{n}$ выбраны так, что при всех $n=0,1, \ldots$

$$
1-\alpha_{n}+\frac{1}{2} \alpha_{n}^{2} h_{n} \leqslant \gamma h_{n},
$$

где $\gamma$ - вещественное число, границы которого уточним ниже. Тогда из (7) и (8) следуют неравенства

$$
\begin{gathered}
\eta_{n+1} \leqslant \gamma \frac{h_{n}}{1-a_{n} h_{n}} \eta_{n}, \\
h_{n+1} \leqslant \gamma \frac{h_{n}^{2}}{\left(1-a_{n} h_{n}\right)^{2}} .
\end{gathered}
$$

Повторное применение неравенства (16) дает

$$
\gamma h_{k} \leqslant \frac{\left(\gamma h_{0}\right)^{2 k}}{\left(1-a_{k-1} h_{k-1}\right)^{21} \ldots\left(1-a_{0} h_{0}\right)^{2^{k}}} .
$$

На основе соотношения (8) (которое остается в силе) можно проверить, что

$$
N\left(h_{n}\right) \leqslant\left(1-\alpha_{n-1} h_{n-1}\right) N^{2}\left(h_{n-1}\right),
$$

причем имеет место равенство, если $\alpha_{n-1}=1$. Из последнего неравенства получим

$N\left(h_{n}\right) \leqslant\left(1-\alpha_{n-1} h_{n-1}\right)\left(1-\alpha_{n-2} h_{n-2}\right)^{2^{1}} \ldots\left(1-\alpha_{0} h_{0}\right)^{2^{n-1}} N^{2^{n}}\left(h_{0}\right)$.

Повторное применение неравенства (15), а также неравенств (18) и (17), дает

$$
\begin{gathered}
\left\|x^{*}-x_{n}\right\| \leqslant N\left(h_{n}\right) \eta_{n} \leqslant N\left(h_{n}\right) \frac{\eta_{0} \cdot \gamma h_{n-1} \ldots \gamma h_{1} \cdot \gamma h_{0}}{\left(1-a_{n-1} h_{n-1}\right) \ldots\left(1-a_{0} h_{0}\right)} \leqslant \\
\leqslant \frac{N\left(h_{0}\right) \eta_{0}\left[\gamma N\left(h_{0}\right) h_{0}\right]^{2^{n}-1}}{\left(1-a_{n-2} h_{n-2}\right)^{21}-1 \ldots\left(1-a_{0} h_{0}\right)^{2 n-1}-1} \leqslant \\
\leqslant \frac{N^{2}\left(h_{0}\right)}{N\left(h_{n}\right)} \eta_{0}\left(1-\alpha_{n-1} h_{n-1}\right) \ldots\left(1-\alpha_{0} h_{0}\right)\left[\gamma N^{2}\left(h_{0}\right) h_{0}\right]^{2^{n}-1} .
\end{gathered}
$$

Полученная формула обеспечит сходимость при $\gamma N^{2}\left(h_{0}\right) h_{0} \leqslant 1$. Но практически последняя оценка в неравенстве (19) успешно применима лишь тогда, когда $\gamma N^{2}\left(h_{0}\right) h_{0}$ значительно меньше единицы. Только в этом случае можно произведение сомножителей, стоящих вне квадратных скобок, оценить с достаточной точностью (напрнмер, числом $\left.N^{2}\left(h_{0}\right)\left(1-\alpha_{0} h_{0}\right) \eta_{0}\right)$, без вычисления величин $h_{k}(k \geqslant 1)$ по рекуррентной формуле (8). Поэтому представляет интерес (менее точная) формула, которая не содержит величины $h_{k}(k \geqslant 1)$.

Учитывая, что $h_{k} \leqslant h_{k-1}, \alpha_{k} \leqslant 1$ и используя предпоследнюю оценку неравенства (19), получим формулу

$$
\left\|x^{*}-x_{n}\right\| \leqslant N\left(h_{0}\right) \eta_{0}\left(1-h_{0}\right)^{n}\left[\frac{\gamma N\left(h_{0}\right) h_{0}}{1-h_{0}}\right]^{2^{n}-1},
$$

которая применима всегда, если только $\gamma N\left(h_{0}\right) h_{0} \leqslant 1-h_{0}$. 
Легко убедиться, что условия (14) и $\alpha_{n} \leqslant 1$ равносильны условиям

$$
1 \geqslant \alpha_{n} \geqslant \frac{1-\sqrt{1-2 h_{n}\left(1-\gamma h_{n}\right)}}{h_{n}}
$$

и $\gamma \geqslant \frac{1}{2}$. Таким образом, нами доказана

Т еор ем а 2. Если выполнены условия $1^{\circ}-4^{\circ}$ теоремы $А$ и вещественные числа * $\alpha_{n}$ выбраны так, что условие (21) выполнено, причем $\gamma$ удовлетворяет неравенству

$$
\frac{1}{2} \leqslant \gamma \leqslant \frac{1-h_{0}}{N\left(h_{0}\right) h_{0}},
$$

то погрешность приближений, полученных методом (2), можно оценить формулой (20) или, еще точнее, формулой (19); в последнем случае достаточно выполнение неравенства $\frac{1}{2} \leqslant \gamma \leqslant \dot{\leqslant}\left[N^{2}\left(h_{0}\right) h_{0}\right]^{-1}$ вместо (22).

Если $\alpha_{n}=1$ при всех $n$, то в силу соотношений (7) и (8) можем взять $\gamma=\frac{1}{2}$ и вместо неравенств (15) и (16) получим равенства (ср. [1])

$$
\begin{aligned}
& \eta_{n+1}=\frac{1}{2} \frac{h_{n}}{1-h_{n}} \eta_{n}, \\
& h_{n+1}=\frac{1}{2} \frac{h_{n}^{2}}{\left(1-h_{n}\right)^{2}} .
\end{aligned}
$$

\section{Следствием теоремы 2 является}

Те орема 3. Если выполнены условия $1^{\circ}-4^{\circ}$ теоремы $A$, то погрешность приближений, полученных методом Ньютона, можно оценить неравенствами

$$
\begin{gathered}
\left\|x^{*}-x_{n}\right\| \leqslant N\left(\bar{h}_{n}\right)\left\|\Gamma\left(x_{n}\right) P\left(x_{n}\right)\right\| \leqslant N\left(h_{n}\right) \eta_{n}= \\
=\frac{N^{2}\left(h_{0}\right)}{N\left(h_{n}\right)} \eta_{0}\left[\prod_{k=0}^{n-1}\left(1-h_{k}\right)\right] \cdot\left[\frac{1}{2} N^{2}\left(h_{0}\right) h_{0}\right]^{2^{n}-1} \leqslant \\
\leqslant N\left(h_{0}\right) \eta_{0}\left(1-h_{0}\right)^{n}\left[\frac{N\left(h_{0}\right) h_{0}}{2\left(1-h_{0}\right)}\right]^{2^{n}-1},
\end{gathered}
$$

где $\bar{h}_{n}=\left\|\Gamma\left(x_{n}\right)\right\| K\left\|\Gamma\left(x_{n}\right) P\left(x_{n}\right)\right\|, a h_{k}(k=1,2, \ldots)$ определены рекуррентно формулой (23).

Примечание 2. 1) Апостериорную оценку удобнее использовать в виде (13) или, учитывая, что $\left\|P\left(x_{n}\right)\right\| \leqslant \frac{1}{2} K\left\|x_{n}-x_{n-1}\right\|^{2}$, в виде

$$
\left\|x^{*}-x_{n}\right\| \leqslant \frac{1}{2} K\left\|\Gamma\left(x_{n-1}\right)\right\| N^{2}\left(\bar{h}_{n-1}\right) \llbracket x_{n}-x_{n-1} \|^{2},
$$

тде $\bar{h}_{n-1}=\left\|I\left(x_{n-1}\right)\right\| K\left\|\Gamma\left(x_{n-1}\right) P\left(x_{n-1}\right)\right\|$.

* Если вычислим $m$ приближений, то достаточно выбрать только $m$ чисел $\alpha_{n}$ $(n=0,1, \ldots, m-1)$, удовлетворяющих условию (21). Остальнье числа $\alpha_{m}$, $\alpha_{m+1}, \ldots$, которые мы не используем, можно считать равными 1. 
2) Так как

$$
\frac{1}{2} N^{2}\left(h_{0}\right) h_{0} \leqslant \frac{N\left(h_{0}\right) h_{0}}{2\left(1-h_{0}\right)} \leqslant \frac{h_{0}}{2\left(1-h_{0}\right)^{2}} \leqslant 2 h_{0},
$$

то при $h_{0}<\frac{1}{2}$ теорема 3 уточняет оценку, полученную С. Ю. Ульмом [ $\left.{ }^{2}\right]$, и в еще большей степени оценку (3), данную Л. В. Канторовичем [']. Эти уточнения тем значительнее, чем больше $n$ и чем меньше $h_{0}$. Теорема 3 уточняет результат С. Ю. Ульма еще в другом смысле, так как в [2] требуется, чтобы неравенство $\left\|P^{\prime \prime}(x)\right\| \leqslant K^{\prime}$ выполнялось в сфере $\left\|x-x_{0}\right\| \leqslant \frac{1-h_{0}^{\prime}}{1-\frac{3}{2} h_{0}^{\prime}} \eta_{0} \quad\left(h_{0}^{\prime}=B_{0} K^{\prime} \eta_{0}\right)$, которая содержит сферу $S\left(x_{0}, r\right)$.

5. Рассмотрим теперь случай, когда $0<\alpha_{n}<2$, причем $\alpha=\inf _{n} \alpha_{n}>0$ и $1<\beta=\sup _{n} \alpha_{n}<2$ (если $\beta \leqslant 1$, то имеем случай, рассмотренный выше).

Т е о ре м а 4. Пусть выполнены условия:

$1^{\circ}$ существует обратный оператор $\Gamma\left(x_{0}\right)=\left[P^{\prime}\left(x_{0}\right)\right]^{-1}$, причем $\left\|\Gamma\left(x_{0}\right)\right\| \leqslant$ $\leqslant B_{0}$

$2^{\circ}\left\|\Gamma\left(x_{0}\right) P\left(x_{0}\right)\right\| \leqslant \eta_{0} ;$

$3^{\circ}$ для всех $x \in S\left(x_{0}, r\right)$, дде $r=\frac{\beta \eta_{0}}{1-q} \quad u \quad q=\max \left\{\frac{|1-a|+\frac{1}{2} a^{2} h_{0}}{1-\alpha h_{0}}\right.$, $\left.\frac{\beta-1+\frac{1}{2} \beta^{2} h_{0}}{1-\beta h_{0}}\right\}$, имеет место оценка $\left\|P^{\prime \prime}(x)\right\| \leqslant K$;

$4^{\circ}$ справедливы неравенства

$$
\begin{gathered}
|1-\alpha|+\frac{1}{2} \alpha^{2} h_{0} \leqslant\left(1-\alpha h_{0}\right)^{2}, \\
\beta-1+\frac{1}{2} \beta^{2} h_{0} \leqslant\left(1-\beta h_{0}\right)^{2}, \beta h_{0}<1,
\end{gathered}
$$

əде $h_{0}=B_{0} K \eta_{0}$.

Тогда уравнение (1) имеет в сфере $S\left(x_{0}, r\right)$ решение $x^{*}, \kappa$ которому сходится последовательность приближений $x_{n}$, полученных методом (2) (где $\left.0<\alpha_{n}<2\right)$, и имеют место оценки

$$
\left\|x^{*}-x_{n}\right\| \leqslant \frac{\beta}{1-q}\left\|\Gamma\left(x_{n}\right) P\left(x_{n}\right)\right\| \leqslant \frac{\beta \eta_{0}}{1-q} q^{n} .
$$

Док а з ательство. Так же как при доказательстве теоремы 1 , можно легко проверить, что: ная $B_{n+1}$, что

$$
\left\|\Gamma\left(x_{n+1}\right)\right\| \leqslant B_{n+1}=\frac{B_{n}}{1-a_{n} h_{n}}
$$


2) найдется постоянная $\eta_{n+1}$, такая, что

$$
\left\|\Gamma\left(x_{n+1}\right) P\left(x_{n+1}\right)\right\| \leqslant \eta_{n+1}=\frac{\left|1-a_{n}\right|+\frac{1}{2} a_{n}^{2} h_{n}}{1-a_{n} h_{n}} \eta_{n} \leqslant q \eta_{n}
$$

3) для всех $x \in S\left(x_{n+1}, r_{n+1}\right)$, где $r_{n+1}=\frac{\beta \eta_{n+1}}{1-q_{n+1}}$ и $q_{n+1}=$

$=\max \left\{\frac{|1-\alpha|+\frac{1}{2} a^{2} h_{n+1}}{1-a h_{n+1}}, \frac{\beta-1+\frac{1}{2} \beta^{2} h_{n+1}}{1-\beta h_{n+1}}\right\} \leqslant q$, имеет место неравенство $\left\|P^{\prime \prime}(x)\right\| \leqslant K$, так как $S\left(x_{n+1}, r_{n+1}\right) \subset S\left(x_{0}, r\right)$;

4) имеют место неравенства, приведенные в условии $4^{\circ}$, где $h_{0}$ заменено на $h_{n+1}$, так как в силу тех же неравенств при индексе $n$

$$
h_{n+1}=B_{n+1} K \eta_{n+1}=\frac{\left|1-a_{n}\right|+\frac{1}{2} a_{n}^{2} h_{n}}{\left(1-\alpha_{n} h_{n}\right)^{2}} h_{n} \leqslant h_{n}
$$

Таким образом, условия теоремы остаются в силе для всех $x_{n+1}$, найденных по формуле (2).

Теперь получаем оценку

$$
\begin{gathered}
\left\|x^{*}-x_{n}\right\| \leqslant \alpha_{n} \eta_{n}+\alpha_{n+1} \eta_{n+1}+\ldots \leqslant \\
\leqslant \beta \eta_{n}\left(1+q+q^{2}+\ldots\right)=\frac{\beta \eta_{n}}{1-q} \leqslant \frac{\beta \eta_{0}}{1-q} q^{n} .
\end{gathered}
$$

Правильность апостериорной оценки очевидна. Так же как при доказательстве теоремы 1 , можно показать, что $x^{*}$ - решение уравнения (1).

П римечание 3 . Можно доказать и теорему, аналогичную теореме 2, т. е. в том случае, когда $\alpha_{n} \in(0,2)$, сходимость метода (2) может быть также второго порядка.

6. Пусть теперь известна граница нормы оператора $\Gamma(x)$ в целой области (ср. [ [ $\left.{ }^{3,4]}\right)$. Если $0<\alpha_{n} \leqslant 1, \alpha=\inf \alpha_{n}>0, \beta=\sup \alpha_{n}$, то имеет место

Т еорем а 5. Пусть выполнены условия:

$1^{\circ}\left\|P\left(x_{0}\right)\right\| \leqslant \delta_{0}$;

$2^{\circ}$ для всех $x=S\left(x_{0}, r\right)$, где $r=\frac{\beta P \delta_{0}}{1-q}$, существует оператор $\Gamma(x)$, причем $\|\Gamma(x)\| \leqslant B$;

$3^{\circ}$ для всех $x \in S\left(x_{0}, r\right) \quad\left\|P^{\prime \prime}(x)\right\| \leqslant K$;

$4^{\circ} \beta h_{0}=\beta B^{2} K \delta_{0}<2$.

Тогда уравнение (1) имеет в сфере $S\left(x_{0}, r\right)$ решение $x^{*}, \kappa$ которому сходится последовательность приближений $x_{n}$, полученных методом (2), и имеет место оценка

$$
\left\|x^{*}-x_{n}\right\| \leqslant \frac{\beta B}{1-q}\left\|P\left(x_{n}\right)\right\| \leqslant \frac{\beta B \delta_{0}}{1-q} \cdot q^{n},
$$

где $q=\max \left\{1-\alpha+\frac{1}{2} \alpha^{2} h_{0}, 1-\beta+\frac{1}{2} \beta^{2} h_{0}\right\}$. 
Д ок аз а тельст в о. Из формулы Тейлора для операторов и из соотношения (2) следует, что

$$
P\left(x_{n+1}\right)=\left(1-\alpha_{n}\right) P\left(x_{n}\right)+R_{n},
$$

где $\left\|R_{n}\right\| \leqslant \frac{1}{2}\left\|P^{\prime \prime}\left(x_{n}+\tau_{n}\left(x_{n+1}-x_{n}\right)\right)\right\|\left\|x_{n+1}-x_{n}\right\|^{2}, 0 \leqslant \tau_{n} \leqslant 1$. Так как $x_{1} \in S\left(x_{0}, r\right)$ и $q<1$ (в силу $4^{\circ}$ ), то из тождества (24) следуют неравенства

$$
\left\|P\left(x_{1}\right)\right\| \leqslant \delta_{1}=\left(1-\alpha_{0}+\frac{1}{2} \alpha_{0}^{2} h_{0}\right) \delta_{0} \leqslant q \delta_{0}<\delta_{0} .
$$

Отсюда имеем

$$
\beta h_{1}=\beta B^{2} K \delta_{1}<\beta B^{2} K \delta_{0} \leq 2 .
$$

Легко проверить, что $S\left(x_{1}, r_{1}\right) \subset S\left(x_{0}, r\right)$, где $r_{1}=\frac{\beta B \delta_{1}}{1-q_{1}}$ и $q_{1}=\max \left\{1-\alpha+\frac{1}{2} \alpha^{2} h_{1}, 1-\beta+\frac{1}{2} \beta^{2} h_{1}\right\} \leqslant q$. Поэтому, если $x_{0}$ заменить на $x_{1}$, то оценки $3^{\circ}$ и $4^{\circ}$ можно оставить прежними. Это значит, что все условия теоремы выполнены, если $x_{0}$ заменить на $x_{1}$, а постоянные $\delta_{0}$, $h_{0}, r$ на $\delta_{1}, h_{1}, r_{1}$ (соответственно). Продолжая процесс, получим оценки

$$
\left\|P\left(x_{n+1}\right)\right\| \leqslant \delta_{n+1}=\left(1-\alpha_{n}+\frac{1}{2} a_{n}^{2} h_{n}\right) \delta_{n} \leqslant q \delta_{n} .
$$

Заключительная часть доказательства очевидна, если иметь в виду доказательства предыдущих теорем.

Пр и мечание 4 . Если $\alpha_{n}=1$ для всех $n$, то предположения теоремы 5 можно несколько ослабить (см. [4]).

Если $\alpha_{n}=\alpha \leqslant 1$ для всех $n$, то $q=1-\alpha+\frac{1}{2} \alpha^{2} h_{0}$ и радиус $r=\frac{a}{1-q} B \delta_{0}=\frac{B \delta_{0}}{1-\frac{1}{2} a h_{0}}$ сферы $S\left(x_{0}, r\right)$ уменьшается с уменьшением $\alpha$. Пусть условия $2^{\circ}$ и $3^{\circ}$ теоремы 5 выполнены для всех элементов $x$ некоторой сферы $S\left(x_{0}, R\right)$. Выбирая $\alpha$ достаточно малым, можно всегда достигнуть того, что $\alpha h_{0}<2$ и $r \equiv r(\alpha) \leqslant R$, если только $R>B \delta_{0}$. Таким образом, можем сформулировать следующую теорему:

Т е о рем а 6. Если в некоторой сфере $S\left(x_{0}, R\right)$, где

$$
R>B\left\|P\left(x_{0}\right)\right\| \text {, }
$$

существует обратный оператор $\Gamma(x)=\left[P^{\prime}(x)\right]^{-1}$, норма которого ограничена числом $B$

$$
\|\Gamma(x)\| \leqslant B,
$$

и $\left\|P^{\prime \prime}(x)\right\|$ также ограничена в сфере $S\left(x_{0}, R\right)$, то уравнение (1) имеет в этой сфере решение , причем сходящиеся $\kappa$ этому решению приближения можно вычислить методом (2), где $\alpha_{n}=\alpha u \alpha$-достаточно мапое положительное число.

При мечание 5 . Можно доказать сходимость метода (2) также и в том случае, когда $\alpha_{n} \in(0,2)$ (и граница нормы $\Gamma(x)$ найдена в целой области) (ср. теорему 4). При выполнении некоторых дополнитель-

- При более слабых предположениях существование решения уравнения (1) доказал М. К. Гавурин[6], но у нас более существенной является вторая половина утверждения. 
ных условий сходимость этого метода - второго порядка (ср. теоремы 2 и 3).

7. Рассмотрим, наконец, еще более общий метод

$$
x_{n+1}=x_{n}-A_{n} \Gamma\left(x_{n}\right) P\left(x_{n}\right),
$$

где $A_{n}$ - произвольные линейные операторы из пространства $X$ в то же пространство. Если, в частности, $A_{n}=\alpha_{n} E$, где $\alpha_{n} \in(0,2)$, то получим метод (2).

Пусть известны оценки *

$$
\left\|E-A_{n}\right\| \leqslant e_{n}, \quad\left\|A_{n}\right\| \leqslant a_{n},
$$

причем $e_{n}$ удовлетворяют условию

$$
e_{n} \leqslant e<1
$$

Пользуясь формулой Тейлора и тождеством

получим

$$
P\left(x_{n}\right)=P^{\prime}\left(x_{n}\right) \Gamma\left(x_{n}\right) P\left(x_{n}\right),
$$

$$
P\left(x_{n+1}\right)=P^{\prime}\left(x_{n}\right)\left(E-A_{n}\right) \Gamma\left(x_{n}\right) P\left(x_{n}\right)+R_{n}
$$

и отсюда

$$
\Gamma\left(x_{n+1}\right) P\left(x_{n+1}\right)=H_{n}\left(E-A_{n}\right) I\left(x_{n}\right) P\left(x_{n}\right)+\Gamma\left(x_{n+1}\right) R_{n},
$$

где $\left\|R_{n}\right\| \leqslant \frac{1}{2}\left\|P^{\prime \prime}\left(x_{n}+\tau_{n}\left(x_{n+1}-x_{n}\right)\right)\right\|\left\|x_{n+1}-x_{n}\right\|^{2}, \quad 0 \leqslant \tau_{n} \leqslant 1$

и $H_{n}=\left[E-\Gamma\left(x_{n}\right)\left(P^{\prime}\left(x_{n}\right)-P^{\prime}\left(x_{n+1}\right)\right)\right]^{-1}$.

Используя тождество (29) и методику доказательства предыдущих теорем, легко получить нижеследующие теоремы:

Т ео рем а 7. Пусть выполнены условия:

$1^{\circ}$ существует обратный оператор $\Gamma\left(x_{0}\right)=\left[P^{\prime}\left(x_{0}\right)\right]^{-1}, \quad$ причем $\left\|\Gamma\left(x_{0}\right)\right\| \leqslant B_{0}$

$2^{\circ} \quad\left\|\Gamma\left(x_{0}\right) P\left(x_{0}\right)\right\| \leqslant \eta_{0} ;$

$3^{\circ}$ для всех $x \in S\left(x_{0}, r\right)$, где $r=\frac{b \eta_{0}}{1-q}$, имеет место оценка $\left\|P^{\prime \prime}(x)\right\| \leqslant K$;

$4^{\circ}$ имеет место неравенстео

$$
e+\frac{1}{2} b^{2} h_{0} \leqslant\left(1-b h_{0}\right)^{2}
$$

где $b h_{0}=b B_{0} K \eta_{0}<1, b=\sup a_{n}<2, \quad q=\frac{e+\frac{1}{2} b^{2} h_{0}}{1-b h_{0}}, a$ a $a_{n}$ e onpeдeлены неравенствами (26) и (27).

Тогда уравнение (1) имеет в сфере $S\left(x_{0}, r\right)$ решение $x^{*}, \kappa$ которому сходится последовательность приближений $x_{n}$, полученных методом (25), и имеют место оценки

$$
\left\|x^{*}-x_{n}\right\| \leqslant \frac{b}{1-q}\left\|\Gamma\left(x_{n}\right) P\left(x_{n}\right)\right\| \leqslant \frac{b \eta_{0}}{1-q} \cdot q^{n} .
$$

- Так как $\left\|A_{n}\right\| \leqslant 1+\left\|E-A_{n}\right\|$, то можно предположить, что $a_{n} \leqslant 1+e_{n}$. 
Т еорем а 8. Пусть выполнены условия:

$1^{\circ}$ существует обратный оператор $\Gamma\left(x_{0}\right)=\left[P^{\prime}\left(x_{0}\right)\right]^{-1}, \quad$ причем $\left\|\Gamma\left(x_{0}\right)\right\| \leqslant B_{0}$

$2^{\circ}\left\|\Gamma\left(x_{0}\right) P\left(x_{0}\right)\right\| \leqslant \eta_{0} ;$

$3^{\circ}$ для всех $x \in S\left(x_{0}, r\right)$, где $r=\frac{b \eta_{0}}{1-\left(1-b h_{0}\right) l_{0}}$, имеет место неравенство $\left\|P^{\prime \prime}(x)\right\| \leqslant K$;

$4^{\circ}$ операторы $A_{n}$ выбраны так, что при всех $n$

$$
e_{n}+\frac{1}{2} a_{n}^{2} h_{n} \leqslant \gamma h_{n},
$$

где ү удовлетворяет условию

$$
\frac{1}{2} \leqslant \gamma \leqslant \frac{\left(1-b h_{0}\right)^{2}}{h_{0}},
$$

a $b h_{0}=b B_{0} K \eta_{0}<1 u h_{n}(n \geqslant 1)$ определены рекуррентно соотношением

$$
h_{n}=\frac{e_{n-1}+\frac{1}{2} a_{n-1}^{2} h_{n-1}}{\left(1-a_{n-1} h_{n-1}\right)^{2}} h_{n-1} .
$$

Тогда уравнение (1) имеет в сфере $S\left(x_{0}, r\right)$ решение $x^{*}, \kappa$ которому сходится последовательность приближении $x_{n}$, полученных методом (25), и имеет место оценка погрешностей

$$
\left\|x^{*}-x_{n}\right\| \leqslant \frac{b \eta_{0}\left(1-b h_{0}\right)^{n}}{1-\left(1-b h_{0}\right) l_{0}^{2^{n}}} \cdot l_{0}^{2^{n}-1}
$$

где $l_{0}=\frac{\gamma h_{0}}{\left(1-b h_{0}\right)^{2}} \quad\left(b=\sup a_{n}\right)$.

При мечание 6. В случае метода Ньютона (т. е. в том случае, когда $A_{n}=E$ при всех $n$ ) можем выбрать $e_{n}=0, a_{n}=1, \quad b=1$ и $\gamma=\frac{1}{2}$. Так как тогда условие $4^{\circ}$ равносильно условию $h_{0} \leqslant \frac{1}{2}$, то из теоремы 8 следует теорема, полученная С. Ю. Ульмом в работе [2].

При мечание 7 . Если в формуле (25) линейные операторы $A_{n}$ не произвольны, а составлены известным образом из операторов $E$, $\Gamma\left(x_{n}\right) \cdot P^{\prime \prime}\left(x_{n}\right), \ldots, \Gamma\left(x_{n}\right) P^{(k-1)}\left(x_{n}\right)$ и элемента $\Gamma\left(x_{n}\right) P\left(x_{n}\right), \quad$ то метод (25) может иметь сходимость порядка $k$ (см. [ $\left.\left.{ }^{5}\right]\right)$.

Примечание 8. Применение методов (2) и (25) можно истолковать как прибли женн ое вычисление последовательных приближений к решению уравнения (1) при помощи метода Ньютона

$$
x_{n+1}=x_{n}-\Gamma\left(x_{n}\right) P\left(x_{n}\right) .
$$

Таким образом, если последовательные приближения $x_{n+1}$ вычислены приближенно в том смысле, что вместо приращения - $\Gamma\left(x_{n}\right) P\left(x_{n}\right)$ найден некоторый другой элемент $-\alpha_{n} \Gamma\left(x_{n}\right) P\left(x_{n}\right)$ или $-A_{n} \Gamma\left(x_{n}\right) P\left(x_{n}\right)$, где соответственно $\alpha_{n} \in(0,2)$ или $\left\|E-A_{n}\right\| \leqslant e<1$, то полученные при- 
ближения все-таки сходятся к точному решению (теоремы $1,4,5,6,7$ ) . Если допущенная при этом ошибка не слишком велика, то сходимость будет по-прежнему второго порядка (теоремы 2,8 ).

\section{Л И ТЕ Р А Т Р А}

1. Л. В. К а н торович, О методе Ньютона, Тр. Матем. ин-та им. В. А. Стеклова, $28,1949,104-144$.

2. С. Ю. Ульм, О сходимости некоторых итерационных процессов в пространстве Банаха, Уч. зап. Тартуск. гос. ун-та, 42, 1956, 135-142.

3. И. П. Мы с ов ских, К вопросу о сходимости метода Ньютона, Тр. Матем. ин-та им. В. А. Стеклова, 28, 1949, 145-147.

4. И. П. М ысов к и х, О сходимости метода Л. В. Канторовича для решения нелинейных функциональных уравнений и его применениях, Вестн. Ленингр. ун-та, № $11,1953,25-48$.

5. Ю. Я. К а а и к, О сходимости итерационных методов, Уч. зап. Тартуск. гос. ун-та, $62,1958,80-98$.

6. М. К. Г а вурин, Нелинейные функциональные уравнения и непрерывные аналоги итеративных методов, Изв. высш. учебн. заведений. Математика, № 5. $1958,18-31$.

Институт кибернетики

Академии наук Эстонской ССР
Поступнла в редакцию

10. II 1960

\section{UHEST NEWTONI MEETODI OLDISTUSEST}

\section{Kivistik}

Resümee

Käesolevas artiklis käsitletakse operaatorvõrrandi $P(x)=0$ ligikaudset lahendamist meetoditega (2) ja (25). Tõestatakse nende meetoditega saadud lähislahendite jada koonduvus võrrandi $P(x)=0$ täpseks lahendiks. Lähislahendite tàpsuse hindamiseks tuletatakse valemid, mis Newtoni meetodi korral täpsustavad L. V. Kantorovitši [ ${ }^{1}$ ] ja S. Ulmi $\left[{ }^{2}\right]$ poolt antud hinnanguid (teoreem 3 ).

\section{Eesti NSV Teaduste Akadeemia \\ Küberneetika Instituut}

Saabus toimetusse

10. II 1960

\section{ON A GENERALIZATION OF NEWTON'S METHOD}

\section{Kivistik}

\section{Summary}

Let $P(x)=0$ be a non-linear operator equation in Banach space. For solving this equation the present paper considers the iterative methods defined by the formulae (2) and (25). Several theorems about the convergence of these methods and formulae for the error estimate are given. Newton's method is considered as a particular case of the method (2). In this case the formulae for the error estimate are preciser than those given by L. V. Kantorovitch [ $\left.{ }^{1}\right]$ and S. Ulm [ [2] (theorem 3).

Akademy of Sciences of the Estonian S.S.R., Institute of Cybernetics

Received

Febr. 10th, 1960 